\title{
Factores asociados a la condición física relacionada con la salud en escolares de la ciudad de Dosquebradas Risaralda
}

\author{
Brayan E Patiño-Palma1
}

\begin{abstract}
RESUMEN
Introducción. La actividad física es un factor de primera magnitud que influye en la salud y la condición física en los adultos; por otra parte, es un determinante del riesgo de mortalidad cardiovascular cuando no se practica de forma regular, además del aumento de la incidencia de obesidad en edades tempranas en países desarrollados, como consecuencia de factores contextuales como currículos escolares, reglas de los padres relativas a la seguridad, limitaciones del ambiente físico, entre otros.

Objetivo. Describir la condición física relacionada con la salud y sus factores asociados en escolares de la ciudad de Dosquebradas, Risaralda, Colombia.

Materiales y Métodos. Estudio de tipo descriptivo transversal, en el cual se calculó una muestra de 339 participantes, matriculados en instituciones educativas públicas y privadas de nivel básico y medio, entre la edad de 12 y 18 años. Se aplica la versión extendida de la batería ALPHA-fitness para evaluar la condición física y una entrevista semiestructurada con la cual se indagó sobre los determinantes sociales de la salud de interés para el estudio.

Resultados. El $60 \%$ de los escolares evaluados presentaron condición física no saludable en tres de los cuatro componentes que evalúa la batería ALPHA Fitness. Se encuentra significancia estadística $(P<0,05)$ con el componente muscular y motor, destacando que entre menor sea el nivel educativo del padre en los escolares, estos, tienen entre 1 y 3 veces más posibilidades de tener un nivel no saludable.

Conclusiones. Se evidencia una alta prevalencia de condición física no saludable además de como el nivel educativo del padre se relaciona estadísticamente con la condición física evaluada en los escolares de la ciudad de Dosquebradas.

Palabras clave: aptitud física, ejercicio, estudiantes, determinantes sociales de la salud, escolaridad, (DeCS).
\end{abstract}

1 Universidad de Boyacá, Tunja, Colombia

Correspondencia: Brayan E Patiño-Palma. Carrera 2a este N 64-169. Tunja (Boyacá), Colombia. Teléfono: (8) 7452105 Ext: 1204.

Correo electrónico: bepatino@uniboyaca.edu.co

Citar este artículo así:

Patiño-Palma BE. Factores asociados a la condición física relacionada con la salud en escolares de la ciudad de Dosquebradas Risaralda. Revista Investig Salud Univ Boyacá. 2019;6(2): 118-136. doi: https://doi.org/10.24267/23897325.343 


\title{
Factors associated with health-related physical condition in schoolchildren in the city of Dosquebradas Risaralda
}

\begin{abstract}
Introduction. Physical activity is a major factor that influences health and physical condition in adults, being a determinant of cardiovascular mortality risk when not practiced regularly in addition to the increase in the incidence of obesity at an early age in developed countries, as a consequence of contextual factors such as school curricula, parental rules regarding safety, limitations of the physical environment, among others.
\end{abstract}

Objective. Describe the physical condition related to health and its associated factors in schoolchildren in the city of Dosquebradas, Risaralda, Colombia.

Materials and Methods. Cross-sectional descriptive study, in which a sample of 339 participants, enrolled in public and private educational institutions of basic and medium level, between the age of 12 and 18 years was included. The extended version of the ALPHA-fitness battery is applied to assess the physical condition and a semi-structured interview with which the social determinants of health of interest for the study were investigated.

Results. $60 \%$ of the students evaluated presented unhealthy physical condition in three of the four components that the ALPHA Fitness battery evaluates. Statistical significance is found $(P<0.05)$ with the muscle and motor component, highlighting that the lower the father's educational level in schoolchildren, these are between 1 and 3 times more likely to have an unhealthy level.

Conclusions. A high prevalence of unhealthy physical condition is evidenced, in addition to how the educational level of the father is statistically related to the physical condition evaluated in schoolchildren in the city of Dosquebradas.

Keywords: physical fitness, exercise, students, social determinants of health, scholarship, (MeSH). 


\section{Fatores associados à condição física relacionada com a saúde em crianças em idade escolar da cidade de Dosquebradas Risaralda.}

\section{RESUMO}

Introdução. A atividade física é um fator de primeira magnitude que influencia na saúde e a condição física em adultos; por outro lado, é um determinante do risco de mortalidade cardiovascular quando não é praticada regularmente, além do aumento da incidência de obesidade em idades precoces nos países desenvolvidos, como consequência de fatores contextuais como currículos escolares, regras de segurança dos pais, limitações do ambiente físico, entre outros.

Objetivo. Descrever a condição física relacionada com a saúde e seus fatores associados em crianças em idade escolar de Dosquebradas, Risaralda, Colômbia.

Materiais e métodos: Estudo descritivo transversal, no qual a amostra calculada foi de 339 participantes, inscritos em instituições de ensino públicas e privadas de nível básico e intermediário, entre 12 e 18 anos. A versão estendida da bateria de teste do ALPHA-fitness foi aplicada para avaliar a condição física e uma entrevista semiestruturada com a qual são investigados os determinantes sociais da saúde do interesse para o estudo.

Resultados. $060 \%$ das crianças em idade escolar avaliadas apresentaram condição física não saudável em três dos quatro componentes avaliados pelo teste do ALPHA-fitness. Significância estatística ( $P$ $<0,05)$ foi encontrada com o componente muscular e motor, destacando que quanto menor o nível educacional do pai das crianças, estas apresentam uma proporção entre 1 e 3 vezes mais chances de ter um nível não saudável.

Conclusões. Uma elevada prevalência de condição física não saudável é evidente, além de como o nível educacional do pai está estatisticamente relacionado à condição física avaliada em crianças em idade escolar da cidade de Dosquebradas.

Palavras-chave: aptidão física, exercício, estudantes, determinantes sociais da saúde, escolaridade. 


\section{INTRODUCCIÓN}

La infancia y la adolescencia son consideradas primordiales en el desarrollo y fomento del estilo de vida, debido a las diferentes experiencias que se tiene a través de la socialización; de esta forma, se fortalecen conductas importantes en la formación de los hábitos saludables que permanecen en la vida adulta(1). En este sentido, es indudable la importancia que tienen los padres en el proceso educativo de todo niño y los diferentes contextos sociales que influyen en la vida diaria de los infantes (2).

Se ha observado como los padres con mayor educación crean ambientes intelectualmente más estimulantes para sus hijos (3-5); de igual manera, Villaseñor et al.(5) concluyen que los hijos de padres con mayor escolaridad muestran mayor desarrollo en la atención y en la memoria.

En lo que respecta al desarrollo de la condición física o práctica de actividad física, Torrijo et al. (6), al igual que Muñoz et al. (7), concluyen que el nivel de estudio de los padres no es un factor determinante del nivel de condición física de sus hijos, sin embargo, resalta las diferencias encontradas entre hombres y mujeres, ya que los varones obtuvieron mejores niveles de condición física en comparación con los escolares de género femenino, lo anterior, debido a que los hombres son más propensos a participar en actividades deportivas, fortaleciendo de esta manera los estereotipos existentes con respecto a la práctica de las actividades físico-deportivas.

Lo anterior, coincide con el estudio de Moya et al. (8), debido a que los deportes practicados en el medio natural son más comunes en el género masculino $(40,5 \%)$ que en el género femenino (25\%), con lo cual se fortalece el estereotipo de que las actividades deportivas son practicadas más por los hombres que por mujeres (9).

Sin embargo, Dundas et al. (10), menciona cómo la exposición de los niños a los entornos en los que desarrollan su vida cotidiana en edades tempranas influye directamente en la salud y en su estilo de vida durante la etapa adulta; asimismo, da a entender que los entornos familiar y escolar, incluso el área residencial, influyen en las características específicas de la vida de los niños, potenciando y desarrollando un nivel de condición física saludable.

En este sentido, la actividad física es un factor de gran magnitud que influye en la salud y la condición física en los adultos, como también es un determinante del riesgo de mortalidad cardiovascular cuando no se practica de forma regular (11-13). En lo que respecta a los escolares, como consecuencia de factores contextuales tales como los currículos escolares, reglas de los padres relativas a la seguridad, limitaciones del ambiente 
físico, entre otras $(14,15)$, estimulan el aumento de la incidencia de obesidad en edades tempranas en países desarrollados $(16,17)$.

El fomento de estos hábitos saludables durante la infancia mejora la esperanza de vida en la edad adulta, demostrando que el nivel de la condición física de los sujetos en edades tempranas se ve reflejado en sus procesos motores en edades adultas (18-20). Lo anterior, permite plantear la relevancia de un buen desarrollo de la condición física desde temprana edad para mejorar, de manera significativa, el desarrollo motor de los niños $(21,22)$.

En la actualidad, se observa el marcado interés en evaluar la condición física de la población escolar, lo que ha motivado a la realización de diversas investigaciones a nivel mundial para proponer baterías de test físicos para evaluar el complejo rendimiento motor (23) y todas las funciones, además de las estructuras que intervienen en la realización de la actividad física o ejercicio. Tales antecedentes promueven las investigaciones en este campo a nivel nacional (24).

Por lo anterior, la presente investigación tiene como objetivo general describir la condición física relacionada con la salud y sus factores asociados en escolares de la ciudad de Dosquebradas, Risaralda, Colombia.

\section{MATERIALES Y MÉTODOS}

La presente investigación es de tipo descriptivo con un diseño transversal, con enfoque cuantitativo y con una fase asociativa. La población consta de 12132 escolares entre los 12 y 18 años, matriculados en los colegios públicos (10850) y privados (1282), de la zona urbana de la ciudad de Dosquebradas Risaralda para el año 2018; según la Secretaría de Educación Municipal, para ese entonces el total de escolares de los colegios públicos en las edades objeto del estudio era de 10850 estudiantes y 1282 para los privados. La muestra fue calculada a través de un diseño probabilístico, por fijación proporcional, teniendo en cuenta para el tamaño muestral el indicador de García et al. (25), los cuales determinaron una prevalencia de Sobrepeso/obesidad de un 32,3\% en escolares. El cálculo de la muestra se realizó por medio del programa Epidat 4.2, con un nivel de confianza del 95\%, determinando así una muestra de 339 participantes, con un porcentaje de pérdida del $10 \%$.

Dentro de los criterios de selección, los participantes debían estar matriculados en una institución educativa (privada o pública), en edades entre 12 y 18 años y aceptar la participación voluntaria en el estudio. Como criterios de exclusión se determinaron aquellos estudiantes que presentaran alteraciones cognitivas, alteraciones metabólicas, embarazo o enfermedades pulmonares. 
Inicialmente, se diligenció una encuesta semiestructurada sobre variables sociodemográficas y/o determinantes de interés; posteriormente, se aplicó la batería ALPHA-fitness para evaluar la condición física de la muestra, batería que fue diseñada a partir de un conjunto de pruebas de campo teniendo en cuenta criterios de validez, fiabilidad, seguridad y viabilidad, para evaluar la condición física con el objetivo principal de orientar trabajos hacia la mejora de la salud de individuos en edades tempranas. Lo anterior, se resume en la figura 1 (26).

Para el desarrollo de la presente investigación se utilizaron tanto la versión extendida de la batería ALPHA-Fitness como los valores de referencia descritos por Ruiz et al. (27) y Secchi et al. $(28,29)$, a partir de los cuales se establecieron como saludable y/o no saludables los diferentes componentes de la batería.

El Alpha-Fitness incluye valoración del componente morfológico, valorado a través de medidas antropométricas tales como el peso, el cual fue tomado por medio de una báscula marca TANITA, calibrada cada 150 mediciones; la talla valorada por medio de un tallímetro portátil de marca SECA 214; los pliegues cutáneos (tríceps y subescapular) con un adipómetro marca Trimcal 4000 Slim, calibrado cada 100 mediciones; se calculó el índice de masa corporal y porcentaje de grasa a partir de los datos obtenidos, además de la valoración del perímetro de cintura medido por medio de una cinta métrica flexible. Tanto la valoración de aspectos de la aptitud física como la evaluación de la fuerza de prensión manual se hicieron con un dinamómetro digital marca Constant, el cual fue calibrado cada 120 mediciones; además, para el test de velocidad 4 × 10 la medición del tiempo se realizó con un cronometro profesional marca Q\&Q, como se sugiere en Palomino, González y Ramos (30); finalmente, fueron calibrados por el grupo investigador integrado por tres evaluadores (2 fisioterapeutas y un licenciado en educación física), quienes se encargaron de valorar la condición física de los escolares.

La condición física general se obtuvo a partir del resultado obtenido en cada una de las pruebas que evalúan los cuatro componentes de la batería ALPHA-Fitness, teniendo en cuenta lo establecido por Pate et al. (31) en cuanto a la resistencia cardiorrespiratoria, la resistencia muscular y la composición corporal. De acuerdo con los resultados, al igual que en Moro et al. (32), se establece que solo se aceptará un componente no saludable para que la condición física en general se determine como saludable.

Una vez recolectada la información, se procedió a realizar el análisis estadístico a través del programa SPSS, versión 23, con la respectiva depuración y limpieza de los datos. Se calcularon las medidas de tendencia central y de variabilidad o dispersión 
Figura 1. Componentes de la condición física

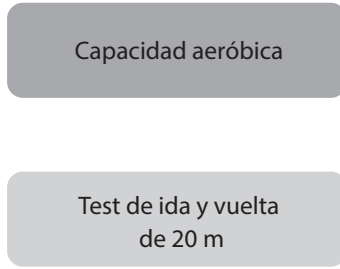
de $20 \mathrm{~m}$
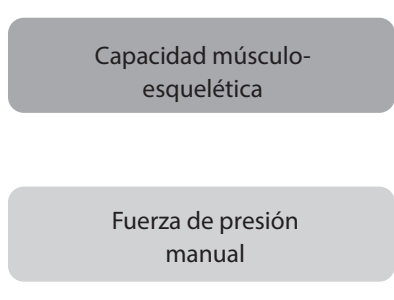

Salto en longitud a pies juntos
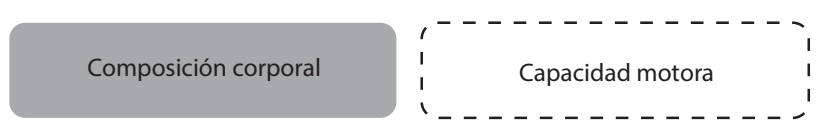

Peso \& altura (IMC)

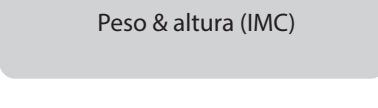

Perímetro de cintura Tríceps \& subescapular para las variables cuantitativas incluidas, además, se permitió de esta manera el análisis descriptivo univariado. El análisis bivariado se desarrolló a partir de las relaciones encontradas entre las variables de estudio y los componentes de la batería Alpha Fitness; posteriormente, se continuó con un análisis mediante un árbol de decisión con el objetivo de encontrar asociaciones entre los determinantes y la condición física general. Para determinar la significancia estadística de las relaciones resultantes, se aplicó la prueba no paramétrica Chi cuadrado con sus respectivos Odds Ratio, con el fin de valorar la intensidad de dichas asociaciones, las cuales son establecidas a partir de las ca- racterísticas propias de las variables categóricas (ordinales y/o nominales).

Una vez seleccionados de forma aleatoria los participantes, se firmaron, por parte de los padres de familia o acudientes, el consentimiento y el asentimiento informado (los cuales fueron aceptados por el comité de ética de la Universidad Autónoma de Manizales mediante el acta número 62 del 15 de febrero de 2017). Tanto la información obtenida como los resultados de la investigación fueron tratados confidencialmente, archivados en papel y medio electrónico. 


\section{RESULTADOS}

Se presentan a continuación los resultados de la evaluación realizada entre enero y septiembre del 2018, aplicada a 339 escolares de colegios públicos y privados de la ciudad de Dosquebradas.

Tabla 1. Distribución de las variables sociodemográficas

\begin{tabular}{cccc}
\hline COLEGIO (Naturaleza) & FRECUENCIA & $\%$ & IC 95\% \\
\hline Privado & 41 & $12,1 \%$ & $8,6-15,5$ \\
\hline Público & 298 & $87,9 \%$ & $84,4-91,3$ \\
\hline SEXO & & & \\
\hline Masculino & 168 & $49,6 \%$ & $44,2-54,8$ \\
\hline Femenino & 171 & $50,4 \%$ & $45,1-55,7$ \\
\hline NIVEL EDUCATIVO & & & \\
\hline $\begin{array}{c}\text { Ninguno- primaria- se- } \\
\text { cundaria }\end{array}$ & 92 & $27,1 \%$ & $22,4-31,8$ \\
\hline $\begin{array}{c}\text { Técnico-tecnológi- } \\
\text { co-universitario }\end{array}$ & 247 & 72,9 & $66,8-76,4$ \\
\hline
\end{tabular}

\begin{tabular}{cccc}
\hline \multicolumn{3}{c}{ NIVEL EDUCATIVO PADRE } & \\
\hline $\begin{array}{c}\text { Ninguno- primaria- se- } \\
\text { cundaria }\end{array}$ & 101 & $29,8 \%$ & $24,9-34,6$ \\
\hline $\begin{array}{c}\text { Técnico-tecnológi- } \\
\text { co-universitario }\end{array}$ & 238 & $70,2 \%$ & $65,3-75,0$ \\
\hline FORMA DE DESPLAZAMIENTO & & \\
\hline${ }^{*}$ Transporte activo & 203 & $59,9 \%$ & $54,6-65,1$ \\
\hline${ }^{*}$ Transporte pasivo & 136 & $40,1 \%$ & $34,9-45,3$ \\
\hline
\end{tabular}

*Transporte activo: caminando y/o bicicleta; **Transporte pasivo: transporte público y/o vehículo familiar (automóvil o moto)

Fuente: autores
En la tabla (1) se observan las diferentes variables independientes incluidas en el estudio, resaltando que más del $80 \%$ de la población evaluada pertenecía a colegios oficiales. Para la variable nivel educativo de los padres, la cual fue reportada por los escolares, prevaleció el nivel educativo entre técnico y universitario, tanto para padres como para madres. Finalmente, se destaca la alta prevalencia del transporte activo que utilizan los escolares para ir al colegio.

Tabla 2. Distribución de las medidas antropométricas

\begin{tabular}{ccccc}
\hline & Promedio & IC 95\% & DS & Max - Min \\
\hline Edad & 14,99 & $14,7-15,21$ & 1,99 & $18-12$ \\
\hline Peso & 54,9 & $53,7-56,0$ & 11,1 & $107,9-28$ \\
\hline Estatura & 162 & $161,0-162,9$ & 8,8 & $187-137$ \\
\hline IMC & 20,8 & $20,4-21,1$ & 3,2 & $30,9-13,7$ \\
\hline $\begin{array}{c}\text { Perímetro de la } \\
\text { cintura }\end{array}$ & 65,2 & $64,8-65,5$ & 7,3 & $91,3-48,5$ \\
\hline $\begin{array}{c}\text { Pliegue tricipital } \\
\text { (mm): }\end{array}$ & 15,4 & $14,7-16,0$ & 6,0 & $54,5-14.2$ \\
\hline $\begin{array}{c}\text { Pliegue subesca- } \\
\text { pular (mm): }\end{array}$ & 13 & $12,4-13,5$ & 5,0 & $48,5-14,6$ \\
\hline \begin{tabular}{c} 
Im \\
\hline
\end{tabular} & & &
\end{tabular}

IMC: Índice de masa corporal; Kg: Kilogramos; Cms: Centímetros; mm: Milímetros; IC 95\%: Intervalo de confianza al 95\%; DS: Desviación estándar Fuente: autores 
Con respecto a las variables antropomórficas, (tabla 2), se destaca que la población escolar de la ciudad de Dosquebradas presenta un peso corporal promedio de 54,9+/-. 11,1 kilogramos y un índice de masa corporal normal según la clasificación de la Organización Mundial de la Salud, contemplada dentro de la resolución 2465 del 2016 (33); finalmente, se resalta el promedio del perímetro de cintura, siendo este de $65,2+/-7,3 \mathrm{~cm}$.

Tabla 3. Componentes de la condición física

\begin{tabular}{|c|c|c|c|}
\hline & FRECUENCIA & $\%$ & IC 95\% \\
\hline \multicolumn{4}{|l|}{ MORFOLÓGICO } \\
\hline Saludable & 20 & 5,9 & $3.392-8.408$ \\
\hline No saludable & 319 & 94,1 & $91.59-96.61$ \\
\hline \multicolumn{4}{|l|}{ MUSCULAR } \\
\hline Saludable & 115 & 33,9 & $28.88-38.96$ \\
\hline No saludable & 224 & 66,1 & $61.04-71.12$ \\
\hline \multicolumn{4}{|l|}{ MOTOR } \\
\hline Saludable & 103 & 30,4 & $25.49-35.28$ \\
\hline No saludable & 236 & 69,6 & 64.7274 .51 \\
\hline \multicolumn{4}{|c|}{ CARDIORESPIRATORIO } \\
\hline Saludable & 178 & 52,5 & $47.19-57.82$ \\
\hline No saludable & 161 & 47,5 & $42.18-52.81$ \\
\hline
\end{tabular}

Fuente: autores
A partir de las pruebas aplicadas por la batería utilizada, se establece el nivel de condición física en relación con la salud, vista a partir de los componentes motor, morfológico, cardiorrespiratorio y muscular. En más del $60 \%$ de los escolares evaluados se encontró condición física no saludable en tres de los cuatro componentes que evalúa la batería ALPHA Fitness (tabla 3).

En la tabla 4. Se nota que el nivel educativo del padre se asocia estadísticamente con el componente muscular, destacando que entre menor sea el nivel educativo del padre en los escolares, los alumnos, tienen entre 1 y 3 veces más posibilidades, como lo indica el intervalo de confianza, de tener un nivel no saludable en mencionado componente.

Con relación al componente motor y al igual que en el componente muscular, únicamente la variable, nivel educativo del padre, muestra diferencia significativa: se destaca la existencia de mayor riesgo de tener un nivel no saludable (entre 1 y 2,9 veces más) con respecto al componente motor, en escolares que refieren tener padres con nivel educativo bajo, en comparación con los escolares que refieren tener padres con nivel educativo alto.

De igual manera, se observa que el tipo de colegio se asocia estadísticamente con el componente motor; en este aspecto se destaca el ser parte de los colegios públicos como un factor protector para obtener un nivel saludable en este componente. 
Tabla 4. Asociaciones entre los determinantes y los componentes condición física.

\begin{tabular}{|c|c|c|c|c|c|c|c|c|c|c|c|c|c|c|c|c|c|c|c|c|}
\hline & \multicolumn{5}{|c|}{ Componente Morfológico } & \multicolumn{5}{|c|}{ Componente Muscular } & \multicolumn{5}{|c|}{ Componente Motor } & \multicolumn{5}{|c|}{ Componente Cardiorrespiratorio } \\
\hline & \multirow{2}{*}{$\begin{array}{l}\text { Saludable } \\
(\%)\end{array}$} & \multirow{2}{*}{$\begin{array}{l}\text { No saludable } \\
(\%)\end{array}$} & \multirow{2}{*}{$\mathrm{P}$ valor } & \multicolumn{2}{|c|}{ OR } & \multirow{2}{*}{$\begin{array}{l}\text { Saludable } \\
(\%)\end{array}$} & \multirow{2}{*}{$\begin{array}{l}\text { No saludable } \\
(\%)\end{array}$} & \multirow{2}{*}{$P$ valor } & \multicolumn{2}{|c|}{ OR } & \multirow{2}{*}{$\begin{array}{l}\text { Saludable } \\
(\%)\end{array}$} & \multirow{2}{*}{$\begin{array}{l}\text { No saludable } \\
(\%)\end{array}$} & \multirow{2}{*}{ P valor } & \multicolumn{2}{|c|}{ OR } & \multirow{2}{*}{$\begin{array}{l}\text { Saludable } \\
(\%)\end{array}$} & \multirow{2}{*}{$\begin{array}{l}\text { No saludable } \\
(\%)\end{array}$} & \multirow{2}{*}{ P valor } & \multicolumn{2}{|c|}{ OR } \\
\hline & & & & Lim inf & Lim sup & & & & Lim inf & Lim sup & & & & Lim inf & Lim sup & & & & Lim inf & Lim sup \\
\hline \multicolumn{21}{|c|}{ Tipo de colegio } \\
\hline Público & 90 & 87,8 & \multirow{2}{*}{0,76} & \multirow{2}{*}{0,28} & \multirow{2}{*}{5,61} & 85,2 & 89,3 & \multirow{2}{*}{0,27} & \multirow{2}{*}{0,35} & \multirow{2}{*}{1,34} & 81,6 & 90,7 & \multirow{2}{*}{${ }^{*} 0,01$} & \multirow{2}{*}{0,23} & \multirow{2}{*}{0,88} & 85,4 & 90,7 & \multirow{2}{*}{0,13} & & \\
\hline Privado & 10 & 12,2 & & & & 14,8 & 10,7 & & & & 18,4 & 9,3 & & & & 14,6 & 9,3 & & 0,30 & 1,11 \\
\hline & & & & & & & & & & dad & & & & & & & & & & \\
\hline 12-14 años & 45 & 42,9 & & & & 43,5 & 42,9 & & & & 44,7 & 42,4 & & & & 39,3 & 47,2 & & & \\
\hline $18-18$ años & 55 & 57,1 & 0,00 & 0,43 & 2,09 & 56,5 & 57,1 & 0,91 & , 03 & 1,011 & 55,3 & 57,6 & 0,09 & 0,68 & 1,12 & 60,7 & 52,8 & 0,14 & 0,47 & 1,1 \\
\hline & & & & & & & & & & exo & & & & & & & & & & \\
\hline Masculino & 65 & 48,6 & & & & 55,7 & 46,4 & & & & 45,6 & 51,3 & & & & 55,1 & 43,5 & & & \\
\hline Femenino & 35 & 51,4 & 0,15 & 0,76 & 5,05 & 44,3 & 53,6 & 0,10 & 0,92 & 2,27 & 54,4 & 48,7 & 0,339 & 0,50 & 1,26 & 44,9 & 56,5 & ${ }^{*} 0,03$ & 1,03 & 2,44 \\
\hline & & & & & & & & & Nivel & ativo madr & & & & & & & & & & \\
\hline $\begin{array}{l}\text { Ninguno Primaria } \\
\text { Secundaria }\end{array}$ & 70 & 73 & & & & 69,6 & 74,6 & & & & 70,9 & 73,7 & & & & 70,8 & 75,2 & & & \\
\hline $\begin{array}{l}\text { Técnico Tecnológico } \\
\text { Universitario }\end{array}$ & 30 & 27 & & & & 30,4 & 25,4 & & & & 29,1 & 26,3 & & & & 29,2 & 24,8 & sol & , & 2,0, \\
\hline
\end{tabular}




\begin{tabular}{|c|c|c|c|c|c|c|c|c|c|c|c|c|c|c|c|c|c|c|c|c|}
\hline & \multicolumn{5}{|c|}{ Componente Morfológico } & \multicolumn{5}{|c|}{ Componente Muscular } & \multicolumn{5}{|c|}{ Componente Motor } & \multicolumn{5}{|c|}{ Componente Cardiorrespiratorio } \\
\hline & \multirow{2}{*}{$\begin{array}{l}\text { Saludable } \\
(\%)\end{array}$} & \multirow{2}{*}{$\begin{array}{l}\text { No saludable } \\
(\%)\end{array}$} & \multirow{2}{*}{ P valor } & \multicolumn{2}{|c|}{ OR } & \multirow{2}{*}{$\begin{array}{c}\text { Saludable } \\
(\%)\end{array}$} & \multirow{2}{*}{$\begin{array}{l}\text { No saludable } \\
(\%)\end{array}$} & \multirow{2}{*}{ P valor } & \multicolumn{2}{|c|}{ OR } & \multirow{2}{*}{$\begin{array}{l}\text { Saludable } \\
(\%)\end{array}$} & \multirow{2}{*}{$\begin{array}{l}\text { No saludable } \\
(\%)\end{array}$} & \multirow{2}{*}{ P valor } & \multicolumn{2}{|c|}{ or } & \multirow{2}{*}{$\begin{array}{l}\text { Saludable } \\
\text { (\%) }\end{array}$} & \multirow{2}{*}{$\begin{array}{l}\text { No saludable } \\
(\%)\end{array}$} & \multirow{2}{*}{ P valor } & \multicolumn{2}{|c|}{ OR } \\
\hline & & & & Lim inf & Lim sup & & & & Lim inf & Lim sup & & & & $\operatorname{Lim} \inf$ & Lim sup & & & & Lim inf & Lim sup \\
\hline \multicolumn{21}{|c|}{ Nivel educativo padre } \\
\hline $\begin{array}{l}\text { Ninguno-primaria- } \\
\text { secundaria }\end{array}$ & 75 & 69,9 & \multirow{2}{*}{0,62} & \multirow{2}{*}{0,27} & \multirow{2}{*}{2,19} & 60,9 & 75 & \multirow{2}{*}{${ }^{*} 0,00$} & \multirow{2}{*}{1,19} & \multirow{2}{*}{3,12} & 61,2 & 74,2 & \multirow{2}{*}{${ }^{*} 0,01$} & \multirow{2}{*}{1,11} & \multirow{2}{*}{2,97} & 66,9 & 26,1 & \multirow{2}{*}{0,15} & \multirow{2}{*}{0,87} & \multirow{2}{*}{2,24} \\
\hline $\begin{array}{l}\text { Técnico tecnológico } \\
\text { Universitario }\end{array}$ & 25 & 30,1 & & & & 39,1 & 25 & & & & 38,8 & 25,8 & & & & 33,1 & 26,1 & & & \\
\hline \multicolumn{21}{|c|}{ Forma de desplazamiento } \\
\hline Activo & 55 & 60.2 & & & & 59,1 & 60,3 & & & & 57,3 & 61 & & & & 63,5 & 55,9 & & & \\
\hline Pasivo & 45 & 39,8 & & & & 40,9 & 39,7 & & 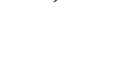 & & 42,7 & 39 & & & & 36,5 & 44,1 & & & 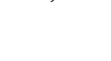 \\
\hline
\end{tabular}

OR: Odds Ratio; Lim inf: Limite Inferior; Lim sup: Límite Superior; * p valor <0,05: Asociación estadísticamente significativa; estadístico de prueba utilizado: Chi cuadrado

Fuente: autores 
Finalmente, en el componente cardiorrespiratorio se aprecia asociación estadísticamente significativa con la variable sexo, observando que las mujeres tienen mayor riesgo (entre 1 y 2,4 veces más) de presentar un rendimiento no saludable en este componente.

Posteriormente, se realiza la codificación de la condición física general, a partir de la evaluación de cada uno de los componentes que evalúa la batería Alpha Fitness y se procede a realizar un árbol de decisión binomial (figura 2) (34), el cual muestra que únicamente la variable nivel educativo del padre presenta asociación estadística con la condición física general; resaltando que entre menor sea el nivel educativo del padre mayor la prevalencia de condición física no saludable en los escolares de la ciudad de Dosquebradas.

Figura 2. Árbol de decisión binomial

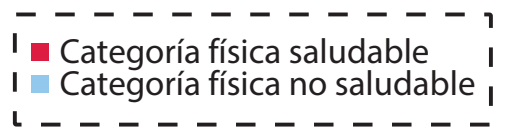

condición física

\begin{tabular}{|lrr|}
\hline Categoría & Nodo 0 & \\
\hline Categoría física saludable & 15,6 & 53 \\
Categoría física no saludable & 84,4 & 286 \\
\hline Total & \multicolumn{4}{|c|}{100,0} & 339 \\
\hline
\end{tabular}

nivel educativo del padre 2

Valor $\mathrm{P}$ corregido $=0,007$, Chi-cuadrado $=7,206 \mathrm{df}=1$

\begin{tabular}{|c|c|}
\hline Ninguno-Primaria-Secundaria & Técnico-Tecnológico-Universidad \\
\hline $\begin{array}{lll}\text { Categoría } & \text { Nodo } 1 & \%\end{array}$ & $\begin{array}{lll}\text { Categoría } & \text { Nodo } 0 & \% \\
\end{array}$ \\
\hline $\begin{array}{lrr}\text { Categoría física saludable } & 12,2 & 29 \\
\text { Categoría física no saludable } & 87,8 & 209 \\
\end{array}$ & \begin{tabular}{|lll} 
Categoría física saludable & 23,8 & 24 \\
Categoría física no saludable & 76,2 & 77 \\
\end{tabular} \\
\hline $\begin{array}{ll}\text { Total } & 70,2238 \\
\end{array}$ & $\begin{array}{ll}\text { Total } & 29,8101\end{array}$ \\
\hline
\end{tabular}

Fuente: Autor 


\section{DISCUSIÓN}

El análisis de los problemas de la salud con el denominado enfoque de los determinantes sociales y económicos es un tema central en la agenda de la organización mundial de la salud y de los ministerios de salud de diferentes países $(35,36)$, además, es un marco de referencia para la investigación en múltiples áreas de la salud pública y la epidemiología $(37,38)$. En la actualidad, un índice bajo de condición física se considera un fuerte factor predictor de enfermedades no transmisibles y de expectativa de vida, por tanto, mantener un buen estado de forma física es una necesidad fisiológica; asimismo, evaluar la condición física es una necesidad médica (39).

En el presente estudio participaron 339 escolares de la ciudad de Dosquebradas Risaralda, en edades comprendidas entre 12 y 18 años, encontrándose una relación de 1:1 entre hombres y mujeres; también, se concluye que ir al colegio en transporte activo es lo más común entre los escolares $(59,9 \%)$, donde se destaca el caminar como la forma más utilizada en ellos $(56,3 \%)$, dato que muestra cierta similitud al ser comparado con los estudios de Rodríguez et al. (40) y Villa et al. (41), quienes concluyen que el $62,4 \%$ y el $56,8 \%$ de escolares respectivamente se transportan al colegio de manera activa, sin embargo, Piñeros y Pardo (42) en su estudio realizado en cinco ciudades colombianas, reportaron que el $50,3 \%$ del total de escolares evaluados no se trasportan de manera activa hacia las aulas de clases (caminar o ir en bicicleta). Lo anterior, según los autores mencionados, puede estar relacionado con las conductas o hábitos que tienen actualmente los escolares, tales como ver televisión, jugar en computadora o video juegos (42).

Secchi et al. (43) referencian un aumento de los niveles de condición física con el incremento de la edad, igualmente, resaltan mayores niveles de fuerza en miembros inferiores en los niños con capacidad aeróbica saludable, un menor índice de masa corporal y perímetro de cintura; sumado a esto, exponen una menor prevalencia del sobrepeso y la obesidad en el grupo con capacidad aeróbica saludable: $22,2 \%$ vs $75,8 \%$ en los participantes masculinos $(p=0,001)$ y $32,2 \%$ vs $57,9 \%$ en los participantes femeninos $(p=0,027)$. En este mismo sentido, en otro estudio (29) se menciona que los adolescentes con capacidad aeróbica saludable tuvieron mayores niveles de fuerza de miembros inferiores, velocidad/agilidad, un menor IMC, menor perímetro de cintura (solo en las mujeres) y menor prevalencia de sobrepeso y obesidad; finalmente, concluye que la muestra de niños y adolescentes masculinos presenta mayores niveles de condición física y que esta diferencia se incrementa con la edad, situación que presenta cierta similitud con los resultados obtenidos en la población participante en este estudio, a pesar de que los datos obtenidos no se discriminaron por género. 
Por su parte, Cano et al. (44) identificaron los factores que determinan el nivel de actividad física; de esa forma, encontraron que el $74 \%$ de la población practica deporte, hábito que fue más frecuente a mayor nivel de estudios en los familiares $(p=0,013)$, en las clases sociales altas $(p=0,021)$ y en el sexo masculino $(p=0,001)$. El porcentaje de niños que pasaba más de 3 horas diarias en actividades sedentarias tenía significancia estadística $(p=0,01)$ según el nivel educativo de los familiares; cabe destacar que el 52,9\% de los niños que pasaban más de 3 horas en actividades sedentarias pertenecían a familias con o sin estudios primarios. Lo anterior, deja ver una gran similitud con los datos obtenidos en la población evaluada, pues se encontró una estrecha relación entre el nivel educativo del padre y la condición física de los escolares $(p<0,05)$; en este sentido, a partir del OR del modelo realizado, se determinó que un escolar hijo de un padre con un nivel educativo entre ninguno y/o bachillerato tiene 2,24 veces más posibilidades de presentar una condición física no saludable.

En el presente proyecto, el total de la muestra evaluada pertenecía a la zona urbana de la ciudad de Dosquebradas Risaralda, sin embargo, es importante destacar los resultados obtenidos por Torres-Luque et al. (45), estudió que abordó la influencia del entorno donde se habita (rural vs urbano) sobre la condición física en estudiantes de educación primaria, encontrando que la po- blación urbana obtiene valores más bajos en parámetros antropométricos en comparación con la población rural; además, la población rural mostró mejores resultados en los diferentes componentes de la condición física (fuerza, flexibilidad y capacidad aeróbica), con lo cual se concluye que el lugar de residencia se debe tener en cuenta a la hora de aplicar programas de intervención para la promoción de la actividad física. Los datos anteriores son similares a los expuestos por Sánchez y Zagalaz (46), quienes establecen que los alumnos del medio rural presentan valores superiores en todas las variables de condición física respecto a los escolares del medio urbano, sin embargo, en cuanto a composición corporal, el alumnado del medio rural, tanto de manera global como separados por sexo, presenta mayores valores de IMC, ratio de cintura-cadera y menores tallas que los del centro urbano. Estos resultados crean una nueva línea de investigación para futuros estudios en la población escolar de ciudad de Dosquebradas y/o departamento de Risaralda.

En este sentido, es necesario insistir en estrategias que mejoren la práctica de actividad física en esta población. La actividad física es una conducta y la condición física es un estado, por tanto, se deben promover conductas activas con el fin de modificar ese estado de condición física. De igual manera, es conveniente establecer programas de prevención basados en la educación y eliminación de los factores que predisponen una 
condición física no saludable, disminuyendo así la prevalencia de enfermedades no transmisibles en edades tempranas. Igualmente, estos resultados deben convertirse en piezas importantes para mejorar los niveles de condición física en la población escolar de Dosquebradas y, desde ahí, presentar recomendaciones como el establecimiento equipos deportivos en los colegios, aumento de las horas de educación física como asignatura, capacitación dentro de la escuela de padres acerca de la promoción de la actividad física y prevención de enfermedades a través del control de factores de riesgo.

Es indispensable que este tipo de estudios continúen, teniendo en cuenta otras variables tales como: el lugar de residencia, mencionada anteriormente; los estudios de tipo longitudinal, y buscar asociaciones o relaciones en otras etapas del ciclo vital.

\section{AGRADECIMIENTOS}

Los autores agradecen a los colegios y escolares de la ciudad de Dosquebradas que participaron en el estudio y quienes permitieron el buen desarrollo de la presente investigación; de igual manera, a la Universidad Autónoma de Manizales y a la Fundación Universitaria Los Libertadores por el apoyo y disposición para llevar a cabo este proyecto.

\section{Limitaciones del estudio}

El método de recolección de los datos pudo limitar la capacidad de análisis de los resultados del presente estudio, pues el referente teórico tenido en cuenta para los puntos de corte no fue representativo para la población colombiana; esto da pie a nuevos objetivos de estudio, ya que en la actualidad no existen valores normativos de la condición física, utilizables como referencia en este tipo de población.

\section{CONFLICTOS DE INTERESES}

El autor declara no tener ningún conflicto de intereses.

\section{FINANCIACIÓN}

El autor declara que la presente investigación no fue financiada ni por entidades públicas ni privadas.

\section{REFERENCIAS}

1. Carrero I, Rupérez E, De Miguel R, Tejero J, Pérez-Gallardo L. Macronutrients intake in school teenagers in Soria capital. Nutr Hosp. 2005;20(3):204-9.

2. Romero S, Garrido M, Zagalaz M. El comportamiento de los padres en el deporte. Retos. 2009;15:29-34. 
3. Hoff E. The Specificity of Environmental Influence: Socioeconomic Status Affects Early Vocabulary Development Via Maternal Speech. Child Dev. 2003;74(5):1368-78. https://doi. org/10.1111/1467-8624.00612

4. Hoff E, Tian C. Socioeconomic status and cultural influences on language. J Commun Disord. 2005 Jul;38(4 SPEC. ISS.):271-8. https://doi. org/10.1016/j.jcomdis.2005.02.003

5. Villaseñor E, Martín A, Díaz E, Rosselli M, Ardila A. Influencia del nivel educativo de los padres, el tipo de escuela y el sexo en el desarrollo de la atención y la memoria. Rev Latinoam Psicol. 2009;41(2):257-76. http:// dx.doi.org/10.14349/rlp.v41ii2.380

6. Torrijo C, Pardo M, Solera M, Gulías R, Amador $S$, Arias N. Relación entre nivel de estudios de los padres y condición física de los escolares: diferencias de género. Rev Andaluza Med del Deport. 2015 Mar;8(1):45-6. http://dx.doi.org/10.1016/j.ramd.2014.10.067

7. Muñoz I, Hernández R, Torres G. Influence of the educational level of the progenitor on the practice of physical activity in children's education students. J Sport Heal Res. 2019;11(2):161-70.

8. Moya I, Ruiz L, Martin J, Ros C. Actividades deportivas en el medio natural: su represen- tación en libros de texto de Educación Física en Primaria. Sport Sci J Sch Sport Phys Educ Psychomot. 2017 Aug 29;3(3):505. Available from: http://hdl.handle.net/2183/22788

9. Morales V, Díaz A, Martínez A, Garcés EJ. Influencia del género en el Deporte Escolar en la Región de Murcia durante el periodo escolar 2005-2010. Sport Sci J Sch Sport Phys Educ Psychomot. 2016 Aug 18;2(3):412. https://doi. org/10.17979/sportis.2016.2.3.1424

10. Dundas R, Leyland AH, MacIntyre S. Early-life school, neighborhood, and family influences on adult health: A multilevel cross-classified analysis of the aberdeen children of the 1950s study. Am J Epidemiol. 2014 Jul 15;180(2):197207. https://doi.org/10.1093/aje/kwu110

11. Muñoz DI, Cardona D, Segura Á, Arango C, Lizcano D. Actividad física recomendada en adultos mayores. Una explicación desde la teoría de los modelos ecológicos. Rev Latinoam Población. 2019 Oct 31;13(25):103-21. https:// doi.org/10.31406/relap2019.v13.i2.n25.5

12. Myers J, Prakash M, Froelicher V, Do D, Partington S, Atwood JE. Exercise Capacity and Mortality among Men Referred for Exercise Testing. N Engl J Med. 2002 Mar 14;346(11):793-801. https://doi.org/10.1056/NEJMoa011858 
13. Kraus W, Houmard J, Duscha B, Knetzger K, Wharton M, McCartney J, et al. Effects of the Amount and Intensity of Exercise on Plasma Lipoproteins. N Engl J Med. 2002 Nov 7;347(19):148392. https://doi.org/10.1056/NEJMoa020194

14. Dollman J, Norton K, Norton L. Evidence for secular trends in children's physical activity behaviour. Br J Sports Med. 2005 Dec 1;39(12):8927. https://doi.org/10.1136/bjsm.2004.016675

15. Orozco E, Pacheco S, Arredondo A, Torres C, Resendiz O. Barreras y facilitadores para una alimentación saludable y actividad física en mujeres embarazadas con sobrepeso y obesidad. Glob Health Promot. 2020;0(0):1-9. https://doi.org/10.1177/1757975920904701

16. Mitchell J, Mattocks C, Ness A, Leary S, Pate R, Dowda M, et al. Sedentary behaviour and obesity in a large cohort of children. Obesity. 2009 Aug;17(8):1596-602. https://doi.org/10.1038/ oby.2009.42

17. Chacín M, Carrillo S, Rodríguez J, Salazar J, Rojas J, Añez R, et al. Obesidad Infantil: Un problema de pequeños que se está volviendo grande Childhood. Rev Latinoam Hipertens. 2019;14(5):616-23.

18. Gómez R. La enseñanza de la Educación Física en el nivel inicial y el primer ciclo de EGB. Buenos Aires: Stadium; 2002.
19. Skinner J, Bounds W, Carruth B, Morris M, Ziegler P. Predictors of children's body mass index: a longitudinal study of diet and growth in children aged 2-8 y. Int J Obes. 2004 Apr 2;28(4):47682. https://doi.org/10.1038/sj.ijo.0802405

20. Alcaldía Mayor de Bogotá. Evaluación de las cualidades físicas en los escolares del Distrito Capital: Aspectos teóricos y metodológicos. Bogotá D.C: Universidad Pedagógica Nacional, editor; 2003.80 p.

21. Sacchetti R, Ceciliani A, Garulli A, Masotti A, Poletti G, Beltrami $P$, et al. Physical fitness of primary school children in relation to overweight prevalence and physical activity habits. J Sports Sci. 2012 Apr;30(7):633-40. https:// doi.org/10.1080/02640414.2012.661070

22. Monteiro M, Amaral T, Oliveira B, Borges N. Protective effect of physical activity on dissatisfaction with body image in children - A cross-sectional study. Psychol Sport Exerc. 2011;12(5):563-9. https://doi.org/10.1016/j. psychsport.2011.05.004

23. Venn A, Thomson R, Schmidt M, Cleland V, Curry B, Gennat $H$, et al. Overweight and obesity from childhood to adulthood: A follow-up of participants in the 1985 Australian Schools Health and Fitness Survey. Med J Aust. 2007;186(9):458-60. 
24. Ramos S, Melo L, Escobar L. Evaluación Antropométrica y motriz condicional de niños y adolescentes de 7 a 18 años. Manizales. Univ caldas. 2007;

\section{García F, Herazo Y, Vidarte JA, García R, Crissien} E. Evaluación de los niveles de actividad física en universitarios mediante método directo. Rev Salud Publica. 2018 Oct 1;20(5):606-11. http://dx.doi.org/10.15446/rsap.v20n5.59003

26. Ruiz J, España V, Castro J, Artero E, Ortega F, García M, et al. Batería ALPHA-Fitness : test de campo para la evaluación de la condición física relacionada con la salud en niños y adolescentes. Nutr Hosp. 2011;26(6):1210-5.

27. R Ruiz J, España V, Castro J, G Artero E, B Ortega $F$, Jiménez $D$, et al. Batería ALPHA-Fitness: test de campo para la evaluación de la condición física relacionada con la salud en niños y adolescentes, manual de instrucciones. Nutr Hosp. 2011;26:1210-4. https://doi. org/10.3305/nh.2011.26.6.5611

28. Secchi J, García G, España-Romero V, Castro-Piñero J. Physical fitness and future cardiovascular risk in argentine children and adolescents: an introduction to the ALPHA test battery. Arch Argent Pediatr. 2014;112(2). https://doi.org/10.5546/aap.2014.132
29. Secchi J, García G, Arcuri C. Evaluación de la condición física relacionada con la salud en el ámbito escolar: un enfoque práctico para interpretar e informar los resultados. Enfoques. 2016;28(2):67-87.

30. Palomino C, González J, Ramos C. Body composition and physical fitness in Colombian secondary school and half of Ibagué. Biomedica. 2017 Sep 1;37(3):408. http://dx.doi. org/10.7705/biomedica.v34i2.3455

31. Pate R, Oria M, Pillsbury L. Fitness Measures and Health Outcomes in Youth. Psysician Sport Med. 1983;11:77-83.

32. Moro $\mathrm{P}$, Castillo $\mathrm{M}$, De Espinosa $\mathrm{M}$, Algaba $\mathrm{E}$, López N, Serrano M. Semilongitudinal analysis of the physical status in madrilenian adolescents. Arch Med del Deport. 2016;33(3):183-92.

33. Ministerio de Salud y Protección Social de Colombia. Resolución 2465. 2016 p. 4-47.

34. Lopera EM. Los árboles de decisión como herramienta para el análisis de riesgos de los proyectos. 2018.

35. OMS. Subsanar las desigualdades en una generación. Informe de la Comisión de Determinantes Sociales en Salud. OMS. 2009. p. 3-5. 
36. Public Health Agency of Canada, World Health Organization. Health Equity Through Intersectoral Action: An Analysis of 18 Country Case Studies. 2008. 40 p.

37. Sobal J, Stunkard AJ. Socioeconomic status and obesity: A review of the literature. Psychol Bull. 1989 Mar;105(2):260-75. https://doi. org/10.1037/0033-2909.105.2.260

38. McLaren L. Socioeconomic status and obesity. Epidemiologic Reviews. 2007;29:29-48. https://doi.org/10.1093/epirev/mxm001

39. Castillo M, Ortega F, Ruiz J. Mejora de la forma física como terapia antienvejecimiento. Med Clin (Barc). 2005;124(4):146-55. https:// dx.doi.org/10.1157/13071011

40. Rodríguez C, Villa E, Pérez I, Delgado M, Ruiz J, Chillón P. Los factores familiares influyen en el desplazamiento activo al colegio de los niños españoles. Nutr Hosp. 2013;28(3):756-63. http://dx.doi.org/10.3305/nh.2013.28.3.6399

41. Villa E, Rodríguez C, Huertas D, Torecedor $P$, Ruiz J, Chillón P. Factores personales y ambientales asociados con el desplazamiento activo al colegio de los escolares españoles. Rev Psicol del Deport. 2011;21(2):343-9.

42. Piñeros M, Pardo C. Actividad física en adolescentes de cinco ciudades colombianas: resul- tados de la Encuesta Mundial de Salud a Escolares. Rev Salud Pública. 2010;12(6):903-14.

43. Secchi J, García G, España V, Castro J. Condición física y riesgo cardiovascular futuro en niños y adolescentes argentinos: una introducción de la batería ALPHA. Arch Argent Pediatr. 2014;112(2):132-40.

44. Cano A, Pérez I, Casares I, Alberola S. Determinantes del nivel de actividad física en escolares y adolescentes: estudio OPACA. An Pediatría. 2011;74(1):15-24. https://doi.org/10.1016/j. anpedi.2010.08.009

45. Torres G, Molero D, Lara A, Latorre P, Cachón J, Zagalaz M. Influencia del entorno donde se habita (rural vs urbano) sobre la condición física de estudiantes de educación primaria. Apunt Med l'Esport. 2014 Oct 1;49(184):105-11. https://doi.org/10.1016/j.apunts.2014.06.005

46. Cruz A, Sánchez A, Zagalaz M, Torres G. Análisis y evaluación de la condición física en estudiantes de educación primaria de un medio rural y urbano. Apunt Educ Fis y Deport. 2014;116(2):44-51. http://dx.doi.org/10.5672/ apunts.2014-0983.es.(2014/2).116.04

Esta obra está bajo una licencia de Creative Commons Reconocimiento-NoComercial 4.0 Internacional 